If we compare the descriptions of gonorrhoea in a textbook of 1928 with those described in a current textbook, the chapters dealing with complications and therapy will be found to be radically changed. Since the introduction of antibiotic therapy, complications have decreased markedly and treatment is almost invariably given systemically.

In our practice we seldom use penicillin because of the risk that it may mask syphilis or provoke allergic reactions, and also because many strains of the gonococcus are developing a progressive resistance. We have found tetracycline, chloramphenicol, streptomycin, rovamycin, kanamycin, and the sulphonamides satisfactory, but at present we are successfully using the 'minute' treatment of gonorrhoea suggested by Siboulet. This consists of the administration of ten tablets of $250 \mathrm{mg}$. thiamphenicol, taken five tablets at a time, with a few minutes interval, on 2 successive days. The results are exceptionally good and we now think that the 'minute' treatment is the therapy of choice for gonococcal infections.

Epidemiological and Therapeutic Data on Gonorrhoea in Genoa and Other Parts of Italy. A. BACCAREDDA-BOY and A. REBORA (Dermosyphilopathic Clinic of the Faculty of Medicine, Genoa, Italy)

This investigation has extended for 13 years, starting from 1958, the year which saw the suppression of brothels and control of prostitution in Italy. The data referring to Genoa show that after the increase observed initially, morbidity dropped and remained practically unchanged.

The same trend was observed for gonorrhoea in the whole region of Liguria. On the other hand, over the whole of Italy as well as in Milan and in Naples, gonorrhoea increased remarkably. An examination of the population curves of Genoa, Naples, and Milan showed that the increase of disease corresponded to a movement of population towards the large towns of people from regions with low standards of hygienic and sexual education.

As far as therapy is concerned, from 1961 to 1970 the incidence of strains with partial resistance to penicillin was 7.63 per cent. in the whole of Italy, while in some cities such as Milan and Trieste the incidence ranged between 40 and 50 per cent.

In the University Dermosyphilopathic Clinic, of Genoa, after the use of thiamphenicol, first used as an experimental drug and later as standard therapy, we saw fewer cases of treatment failure, at the most $4 \cdot 7$ per cent. Other antibiotics have revealed a high percentage (average 20 per cent.) of resistant infections.

Epidemiological Aspects of Gonococcal Infections in Dakar. A. J. FAYE (Dermatological Clinic of the Faculty of Medicine, Dakar, Senegal)

In Senegal, particularly in Dakar, gonococcal infections are more frequent among those of the lower socioeconomic groups who are treated at the Institute of Social Hygiene. The progressive increase of cases does not seem to be related to bacterial resistance to the antibiotics commonly used, but, in Dakar, at least, to a deficiency of health education among our population. Patients neglect their infections or obtain only partial treatment because of their poor economic status and the lack of medicines in our dispensaries.

Changes in habits due to economic and social progress, as well as migration and urbanization, explain the higher occurrence of gonococcal infections in the last few years among young students of from 16 to 18 years of age.

A suitable health education programme would have every prospect of success in limiting the spreading of the disease, since in our country the gonococcus has apparently not yet developed resistance to antibiotics.

Problems Concerning Gonorrhoea in Austria. A. F. LUGER (City Hospital Wien-Lainz, Department of Dermatology, Vienna, Austria)

The number of cases of gonorrhoea in Austria remained approximately the same every year from 1952 to 1969 , whereas cases of syphilis have gradually increased from 1959 onward. A similar tendency with an analogous rate of increase has been observed among the out-patients at the Second University Skin Clinic.

Cases of gonorrhoea are ten to fifty times more frequent among unregistered prostitutes than among registered, controlled prostitutes, whose morbidity rate is not significantly higher than that of the population of Vienna. In the capital the risk of infection with syphilis is greater from casual partners from the general population than from registered prostitutes. The number of cases of gonorrhoea officially reported is highest in March, June, August, and September.

In the years 1960 to 1968 the average age of patients with a first infection fell by $2 \frac{1}{2}$ years.

Penicillin sensitivity of gonococcal strains has not decreased significantly since 1967 . Therapy failures are due mainly to dosage errors or to the administration of delayed absorption preparations.

The intensification of antiepidemic measures should result in a gradual reduction of the problems of gonorrhoea in Austria.

\section{MICROBIOLOGY, DIAGNOSIS and TREATMENT}

\section{FIRST SESSION}

Aspects of the Present Problem of Gonorrhoea in Spain. J. GAY PRIETo (Central University, Department of Dermatology and Venereology, Madrid, Spain)

In Spain, as in many other countries, practitioners and specialists are not obliged to report cases of gonorrhoea, and exact figures of the incidence are not available.

Some important data, recorded by the Epidemiological Unit of the U.S. Sixth Fleet, show that from a venereal point of view the most heavily affected port on the Mediterranean sea is Barcelona ( 7 cases per thousand) followed by Valencia ( 4 cases per thousand), while the incidence in Marseilles has never reached 4 per thousand. Data on gonorrhoea in the well-known holiday areas of Spain confirm the important role of tourism in the spreading of venereal diseases.

Patients treated by general practitioners or specialist physicians or in official venereal disease clinics usually recover after simple antibiotic therapy and we have not 